\title{
PERTURBATIONS OF FRIEDMAN-LEMAITRE-ROBERTSON-WALKER SPACETIMES IN GEROCH-HELD-PENROSE FORMALISM
}

\author{
J.Novák \\ Department of theoretical physics, Charles University, \\ V Holešovičkách 2, Prague-Libeň, 180 00, Czech republic, jan.janno.novak@gmail.com
}

ABSTRACT. We will use Geroch-Held-Penrose formalism for decoupling of quantity $d \Psi_{4}$, which is responsible for tensorial perturbations, in Bianchi equations. We will concentrate on the case, where we eliminate the source terms.

Keywords: Friedman-Lemaitre-Robertson-Walker spacetime, Geroch-Held-Penrose formalism, NewmanPenrose formalism, Weyl scalars

\section{Introduction}

Our goal is to use the Geroch-Held-Penrose formalism (GHP - formalism) in reformulation of perturbations of Friedman-Lemaitre-Robertson-Walker spacetime (FLRW ST). GHP - formalism (a more compact version of NP - formalism) is a convenient formalism, because it allows us to work with partial differential equations of the first order. The scalar and tensor perturbations are for us the most interesting because of the origin of structure. I will show, how to apply the GHP-formalism for decoupling of the quantity $d \psi_{4}$ in Bianchi equation. These calculations are done for the case of the simplified right-hand side (RHS without sources).

\section{NP-formalism}

NP- and GHP-formalisms are mathematical approaches which help us, for example, in perturbation theory to simplify calculations in standard General Relativity. We decompose the metric with respect to the null tetrad and then we project all quantities on this tetrad (in the NP-formalism). The basic quantities are spin-coefficients - projections of the derivatives of the null tetrad, then projections of the Ricci tensor and already mentioned Weyl scalars. We could then rewrite the Einstein's equations by the 18 Ricci, $8+3$ Bianchi and 4 commutation equations, which are only first order PDE's, when we define new derivatives in the direction of the tetrad $(D, \Delta, \delta, \bar{\delta})$. Let us to introduce,
Table 1: Spin coefficients

$$
\begin{array}{lll}
\alpha=\frac{1}{2}\left(S_{214}+S_{344}\right) & \nu=S_{242} & \tau=S_{312} \\
\beta=\frac{1}{2}\left(S_{213}+S_{343}\right) & \kappa=S_{311} & \sigma=S_{313} \\
\gamma=\frac{1}{2}\left(S_{212}+S_{342}\right) & \pi=S_{241} & \mu=S_{243} \\
\epsilon=\frac{1}{2}\left(S_{211}+S_{341}\right) & \rho=S_{314} & \lambda=S_{244}
\end{array}
$$

for illustration, the basic quantities and equations now: we will denote the 12 spin coefficients by $S_{i j k}$ (standard notation is with $\gamma$ ), where the three indices mean, which element of the tetrad we are using (where the null tetrad is defined in standard way), Table 1 .

For example:

$$
\rho=m^{\mu} l_{\mu ; \nu} \bar{m}^{\nu}
$$

Projections of the Ricci tensor (we will omit the brackets by tetrad indices in this part of thesis):

$$
\begin{gathered}
\Phi_{(i)(j)}, i, j=0,1,2,3, \\
\Phi_{(0)(0)}=-\frac{1}{2} R_{\mu \nu} l^{\mu} l^{\nu}
\end{gathered}
$$

So, let's define the projections of the Ricci tensor by the following notation, [1]:

$$
\begin{aligned}
& \Phi_{00}=-\frac{1}{2} R_{11}, \Phi_{01}=-\frac{1}{2} R_{13}, \Phi_{10}=-\frac{1}{2} R_{14}, \\
& \Phi_{12}=-\frac{1}{2} R_{23}, \Phi_{21}=-\frac{1}{2} R_{24}, \Phi_{22}=-\frac{1}{2} R_{22}, \\
& \Phi_{11}=-\frac{1}{4}\left(R_{12}+R_{34}\right), \Lambda=\frac{1}{12}\left(R_{12}-R_{34}\right), \\
& \Phi_{02}=-\frac{1}{2} R_{33}, \Phi_{20}=-\frac{1}{2} R_{44} .
\end{aligned}
$$

Weyl scalars (5 in dimension 4):

$$
\Psi_{i}, \quad i=0,1,2,3,4
$$




$$
\begin{aligned}
& \Psi_{0}=l^{\mu} m^{\nu} l^{\rho} m^{\sigma} C_{\mu \nu \rho \sigma} \\
& \Psi_{1}=l^{\mu} n^{\nu} l^{\rho} m^{\sigma} C_{\mu \nu \rho \sigma} \\
& \Psi_{2}=l^{\mu} m^{\nu} \bar{m}^{\rho} n^{\sigma} C_{\mu \nu \rho \sigma} \\
& \Psi_{3}=n^{\mu} l^{\nu} n^{\rho} \bar{m}^{\sigma} C_{\mu \nu \rho \sigma} \\
& \Psi_{4}=n^{\mu} \bar{m}^{\nu} n^{\rho} \bar{m}^{\sigma} C_{\mu \nu \rho \sigma}
\end{aligned}
$$

Now we present one Ricci, one Bianchi and one commutation relation:

Ricci identities (18 equations)

$D \rho-\delta^{*} \kappa-\rho^{2}-\sigma \bar{\sigma}-\rho \epsilon-\rho \bar{\epsilon}+\bar{\kappa} \tau+(3 \alpha+\bar{\beta}-\pi) \kappa=\Phi_{00}$,

Bianchi identities (11 equations)

$$
\begin{aligned}
& -\delta^{*} \Psi_{0}+D \Psi_{1}+(4 \alpha-\pi) \Psi_{0}-2(2 \rho+\epsilon) \Psi_{1}= \\
& \delta \Phi_{00}-3 \kappa \Psi_{2}-D \Phi_{01}+2(\epsilon+\bar{\rho}) \Phi_{01}-\bar{\kappa} \Phi_{02}+ \\
& +(\bar{\pi}-2 \bar{\alpha}-2 \beta) \Phi_{00}+2 \sigma \Phi_{10}-2 \kappa \Phi_{11}
\end{aligned}
$$

Commutation relations (4 equations):

$\Delta D-D \Delta=(\gamma+\bar{\gamma}) D+(\epsilon+\bar{\epsilon}) \Delta-(\bar{\tau}+\pi) \delta-(\tau+\bar{\pi}) \bar{\delta}$

We can rotate the tetrad and we can get a transformation property of these quantities. However, there exists also a more compact version of the NP-formalism, so called GHP-formalism. One makes the following redefinitions of the derivative operators:

$$
\begin{gathered}
\mathrm{p}=D-p \epsilon-q \bar{\epsilon}, \\
\mathrm{p}^{\prime}=\Delta-p \gamma-q \bar{\gamma}, \\
\partial=\delta-p \beta-q \bar{\alpha} \\
\curvearrowright^{\prime}=\bar{\delta}-p \bar{\beta}-q \alpha .
\end{gathered}
$$

We have 4 different operators and two, so called, dualities in dimension 4 (star - duality, $(p, q) \rightarrow(p,-q)$, is for exchange of the vector $l$ and $m$, the prime duality,
Table 2: Relations among projections of Ricci tensor

$$
\begin{array}{llll}
\Phi_{00}^{\prime}=\Phi_{22} & \Phi_{11}^{\prime}=\Phi_{11} & \Phi_{10}^{\prime}=\Phi_{12} & \Phi_{02}^{\prime}=\Phi_{20} \\
\Phi_{00}^{\prime}=\Phi_{02} & \Phi_{01}^{\prime}=-\Phi_{01} & \Phi_{10}^{*}=\Phi_{12} & \Phi_{11}^{*}=-\Phi_{11} \\
\Phi_{22}^{*}=\Phi_{20} & \Phi_{12}^{*}=\Phi_{10} & \Phi_{02}^{*}=\Phi_{00} & \Phi_{20}^{*}=-\Phi_{22} \\
\Lambda^{\prime}=\Lambda & \Phi_{21}^{*}=-\Phi_{21} & \Lambda^{*}=-\Lambda &
\end{array}
$$

Table 3: GHP-type

$$
\begin{array}{lll}
\Phi_{00}:(2,2) & \Phi_{01}:(2,0) & \Phi_{10}:(0,2) \\
\Phi_{11}:(0,0) & \Phi_{22}:(-2,-2) & \Phi_{12}:(0,-2) \\
\Phi_{21}:(-2,0) & \Phi_{20}:(-2,2) & \Phi_{02}:(2,-2) \\
\Lambda:(0,0) & &
\end{array}
$$

$(p, q) \rightarrow(-p,-q)$, for the exchange of the $l$ and $n$; we could not use both dualities in HD, because we have odd dimensions)

$$
\Sigma \eta, \Sigma=\left\{\mathrm{p}, \mathrm{p}^{\prime}, \mathrm{\partial}, \mathrm{\partial}^{\prime}\right\}
$$

where $\Sigma$ is an operator acting on a quantity $\eta$.

But we need the notion of the GHP scalars when we make the following transformations:

$$
\begin{gathered}
l^{\nu} \rightarrow \lambda^{-1} l^{\nu}, \\
n^{\mu} \rightarrow \lambda n^{\mu}, \\
m^{\rho} \mapsto e^{i \theta} m^{\rho} .
\end{gathered}
$$

We say that a quantity $\eta$ is a GHP-scalar of type $(p, q)$, if it transforms like (analogical definition for the case of higher dimensions is in [2]):

$$
\eta \rightarrow \lambda^{(p+q) / 2} e^{i(p-q) \theta / 2} \eta
$$

We will use the star and prime duality in a standard way, [1]. We see the relations among perturbations of Ricci tensor in Table 2.

And the types for these quantities are in Table 3 .

\section{Computations}

Now we will apply the GHP-formalism in perturbation theory of FLRW ST. It will be done, of course, in classical manner. However, we obtain a new result with this formalism.

Reference [3] will be of great importance for us, where the following fact can be found: the only nonvanishing spin coefficients for the case of FLRW are $\alpha$, $\beta, \gamma, \mu$ and $\rho$. These are the same non-zero spin coefficients as for the case of the Schwarzschild solution. This fact can be employed in the analysis of unperturbed equations. This means that we can get rid of 
many terms in the resulting equations. We get rid of $\alpha, \beta, \gamma$ and $\epsilon$ because they are absorbed into $\mathrm{p}$ and $\partial\left(\mathrm{p}^{\prime}\right.$ and $\left.\mathrm{\partial}^{\prime}\right)$. Together there are 12 spin coefficients, thus there remain yet 8 more: $\tau, \sigma, \kappa, \mu, \rho, \lambda, \pi$ and $\nu$;

The course of action will be now the following. We will write the general Bianchi equations for the case of FLRW ST with sources. We will show our result for the special case of simplified right-hand side.

We have the following 2 equations in GHP formalism for the case of FLRW ST. We have 8 equations in standard NP-formalism, but this formalism is even more efficient. (We stress once again that we have sources on the right hand side of the equations contrary to the Schwarzschild ST.) The equations read

$$
\begin{array}{r}
\mathrm{p} \Psi_{1}-\partial^{\prime} \Psi_{0}+\tau^{\prime} \Psi_{0}-4 \rho \Psi_{1}+3 \kappa \Psi_{2}=\mathrm{p} \Phi_{01}-\partial \Phi_{00}- \\
-\bar{\pi} \Phi_{00}-2 \bar{\rho} \Phi_{01}+\bar{\kappa} \Phi_{02}+2 \kappa \Phi_{11}-2 \sigma \Phi_{10}
\end{array}
$$

$$
\begin{array}{r}
\mathrm{p} \Psi_{2}-\check{\partial}^{\prime} \Psi_{1}-\sigma^{\prime} \Psi_{0}+2 \tau^{\prime} \Psi_{1}-3 \rho \Psi_{2}+2 \kappa \Psi_{3}= \\
\mathrm{p}^{\prime} \Phi_{00}-\partial^{\prime} \Phi_{01}-\bar{\rho}^{\prime} \Phi_{00}+2 \bar{\tau} \Phi_{01}-2 \rho \Phi_{11}- \\
-\bar{\sigma} \Phi_{00}^{*}+2 \tau \Phi_{10}+2 \mathrm{p} \Lambda
\end{array}
$$

where we defined the NP components of the Weyl tensor in the standard way.

The $\Psi_{0}$ and $\Psi_{4}$ are connected with the tensor perturbations, $\Psi_{1}$ and $\Psi_{3}$ are connected with the vector perturbations and $\Psi_{2}$ is connected with the scalar perturbations according to the $[3]^{1} .^{2}$

Now we will follow the approach presented in [4]. The difference, as we already mentioned, is that we have sources on the RHS. However, we can make the same steps: we will take the first equation and we will apply operator $\partial$, we make the star duality and we add the first and this modified equation. Then we plug from the Ricci identities, we eliminate some of these combinations of spin coefficients (we make also prime and star dualities of these Ricci identities ) and we arrive at the following result (the second equation could be obtained in a similar way).

\footnotetext{
${ }^{1}$ We can use their boost weights like an argument.

${ }^{2}$ In the case of non-zero sources we have also other two equations:

$$
\begin{gathered}
-\left[\mathrm{p}^{\prime}-2 \bar{\tau}^{*}+\pi^{*}\right] \Phi_{01}+\left[-\mathrm{p}-2 \tau^{*}+\bar{\pi}^{*}\right] \Phi_{12}+ \\
{\left[\partial-2\left(\rho^{*}+\bar{\rho}^{*}\right] \Phi_{11}-\left[-\widetilde{\partial}^{\prime}+\mu^{*}+\bar{\mu}^{*}\right] \Phi_{02}+\right.} \\
\bar{\sigma}^{*} \Phi_{02}^{*}+\sigma^{*} \Phi_{20}^{*}-\bar{\kappa}^{*} \Phi_{12}^{*}-\kappa^{*} \Phi_{21}^{*}+3 ð \Lambda=0, \\
{\left[\check{\partial}-2 \tau+2 \pi^{*}\right] \Phi_{11}-3 ð \Lambda+[-\mathrm{p}+2 \rho+\bar{\rho}] \Phi_{12}+} \\
{\left[-\mathrm{p}^{\prime}-2 \bar{\mu}-\mu\right] \Phi_{01}+\left[\check{\partial}^{\prime}-\tau^{*}+\pi\right] \Phi_{02}}
\end{gathered}
$$$$
-\kappa \Phi_{22}+\bar{\nu} \Phi_{00}+\sigma \Phi_{21}-\bar{\lambda} \Phi_{10}=0 .
$$

$$
\begin{array}{r}
{\left[\mathrm{p}^{\prime} \mathrm{p}-\check{\partial}^{\prime} \partial-\left(4 \rho^{\prime}+\bar{\rho}^{\prime}\right) \mathrm{p}-\rho \mathrm{p}^{\prime}+\left(4 \tau^{\prime}+\bar{\tau}\right) ð+\right.} \\
\left.\tau \partial^{\prime}+4 \rho \rho^{\prime}-4 \tau \tau^{\prime}-2 \Psi_{2}+2 \Lambda\right] \Psi_{4}+ \\
{\left[4 \mathrm{p} \kappa^{\prime}-4 ð \sigma^{\prime}-4(\bar{\rho}-2 \rho) \kappa^{\prime}+\right.} \\
\left.4(\bar{\tau}-2 \tau) \sigma^{\prime}+10 \Psi_{3}\right] \Psi_{3}+ \\
{\left[-4 \sigma^{\prime} \mathrm{p}^{\prime}+4 \kappa^{\prime} \partial^{\prime}-12 \kappa^{\prime} \tau^{\prime}+12 \rho^{\prime} \sigma^{\prime}-3 \Psi_{0}\right] \Psi_{2}=0 .}
\end{array}
$$

This equation contains information from both (16) and (17). It is interesting that for this case of FLRW spacetimes, we have cancellations of all extra terms in front of $\Psi_{2}$ and $\Psi_{3}$. The terms in the brackets in front of $\Psi_{2}$ and $\Psi_{3}$ are exactly the same (except of one term $3 \Psi_{0} \Psi_{2}$ ) as for the case of the Schwarzschild spacetime. This means that when we will make perturbations of these equations, the second and third term disappear. So, we obtain a decoupling of the quantity $d \Psi_{4}$.

This is other new information, when we compare it with [3]. Here we were interested in equations without sources, i.e. when we put just delta-function on the RHS. But in later work we could be interested in the same problem but with sources, as was already suggested in this article. It is an advantage to write all sources in one compact form.

\section{Conclusion}

I have been studying perturbation theory of FLRW ST in GHP formalism. We obtained a new interesting observation, which could be used for other research in the realm of Cosmological Perturbation Theory.

\section{References}

Chandrasekhar S.: 1983, Mathematical theory of black holes, Oxford University Press, 668.

Durkee M., Pravda V., Pravdová A., Reall H.S.: 2010, Generalization of the Geroch-Held-Penrose formalism to higher dimensions, Class.Quantum Grav., 27.

Sharma S., Khanal U.: 2014, Perturbations of FRW ST's in NP formlism, International Journal of Modern Physics D.

Stewart J.M., Walker M.: 2014, Proc. R.Soc. Lond. A, 49-74. 